\title{
Filigrane
}

Écoutes psychanalytiques

\section{Jeu, trauma, jeu traumatique et capacité de mentalisation chez l'enfant : considérations cliniques et thérapeutiques}

\author{
Miguel M. Terradas, Mélissa Paquette, Cécilanne Lepage-Voyer et Vincent \\ Domon-Archambault
}

Volume 26, numéro 2, 2017

La terreur des enfants : deuxième partie

URI : https://id.erudit.org/iderudit/1055358ar

DOI : https://doi.org/10.7202/1055358ar

Aller au sommaire du numéro

Éditeur(s)

Revue Santé mentale au Québec

ISSN

1192-1412 (imprimé)

1911-4656 (numérique)

Découvrir la revue

Citer cet article

Terradas, M. M., Paquette, M., Lepage-Voyer, C. \& Domon-Archambault, V.

(2017). Jeu, trauma, jeu traumatique et capacité de mentalisation chez l'enfant : considérations cliniques et thérapeutiques. Filigrane, 26(2), 27-44.

https://doi.org/10.7202/1055358ar
Résumé de l'article

Le jeu symbolique et la capacité de mentalisation (CM) constituent des ressources sur lesquelles s'appuie le psychothérapeute pour aider l'enfant à élaborer ses conflits intrapsychiques et ses difficultés relationnelles. Ces capacités ne se développent pas de façon optimale lorsque l'enfant est confronté, dès un très jeune âge, à des traumas complexes, c'est-à-dire à des situations où il est exposé, de façon répétée et prolongée dans le temps, à des événements traumatiques qui surviennent au sein de ses relations significatives. Cet article présente d'abord les éléments essentiels à la compréhension du jeu comme moyen d'expression de l'enfant. Ensuite, les conséquences des traumas complexes sur la capacité à jouer et à faire semblant sont présentées. L'une de celles-ci concerne la présence de jeu traumatique, qui se caractérise par la répétition littérale du trauma. Bien que l'enfant fournisse des efforts pour échapper à l'emprise du trauma, ses jeux sont envahis par des éléments anxiogènes liés à ce dernier. Enfin, les impacts possibles des traumas complexes sur la capacité de mentalisation de l'enfant sont abordés. Les auteurs suggèrent que ce type de trauma altère le développement de cette capacité et engendre chez l'enfant un fonctionnement psychique caractéristique des modes prémentalisants, dont les répercussions peuvent être observées dans son jeu. 


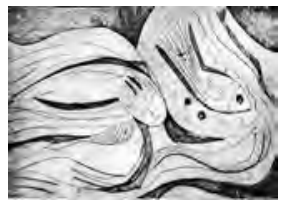

\title{
Jeu, trauma, jeu traumatique et capacité de mentalisation chez l'enfant: considérations cliniques et thérapeutiques
}

\author{
Miguel M.Terradas, \\ Mélissa Paquette, Cécilanne Lepage- \\ Voyer et Vincent Domon- \\ Archambault
}

\begin{abstract}
RÉSUMÉ: Le jeu symbolique et la capacité de mentalisation (CM) constituent des ressources sur lesquelles s'appuie le psychothérapeute pour aider l'enfant à élaborer ses conflits intrapsychiques et ses difficultés relationnelles. Ces capacités ne se développent pas de façon optimale lorsque l'enfant est confronté, dès un très jeune âge, à des traumas complexes, c'est-à-dire à des situations où il est exposé, de façon répétée et prolongée dans le temps, à des événements traumatiques qui surviennent au sein de ses relations significatives. Cet article présente d'abord les éléments essentiels à la compréhension du jeu comme moyen d'expression de l'enfant. Ensuite, les conséquences des traumas complexes sur la capacité à jouer et à faire semblant sont présentées. L'une de celles-ci concerne la présence de jeu traumatique, qui se caractérise par la répétition littérale du trauma. Bien que l'enfant fournisse des efforts pour échapper à l'emprise du trauma, ses jeux sont envahis par des éléments anxiogènes liés à ce dernier. Enfin, les impacts possibles des traumas complexes sur la capacité de mentalisation de l'enfant sont abordés. Les auteurs suggèrent que ce type de trauma altère le développement de cette capacité et engendre chez l'enfant un fonctionnement psychique caractéristique des modes prémentalisants, dont les répercussions peuvent être observées dans son jeu.
\end{abstract}

Mots-clés: jeu, trauma, jeu traumatique, capacité de mentalisation, enfant

\begin{abstract}
Child's symbolic play and mentalizing capacity (MC) are essential resources to work through intrapsychic conflicts and relational difficulties in psychotherapy. However, these abilities are commonly underdeveloped in children who have been exposed, repeatedly and from a very young age, to traumatic events that occur within their significant relationships. First, this article presents the concepts that are fundamental for understanding the role of play as a means of communication. Second, the consequences of complex trauma on the child's ability to use pretend play in psychotherapy are exposed. One of these consequences is
\end{abstract}


related to the presence of traumatic play, which is characterized by the literal repetition of traumatic events. Although the child strives to avoid the outcomes of the trauma, the anxiety linked to it could invade his or her play activities. Finally, the possible impacts of complex traumas on the child's MC are discussed. The authors suggest that children who have been exposed to complex trauma present prementalizing modes of psychic functioning that of which repercussions can be observed in their play activities.

Keywords: play, trauma, post-traumatic pay, mentalizing capacity, child

\section{Le jeu de l'enfant: sa définition et son développement}

D lusieurs auteurs considèrent que le jeu est le médium privilégié pouvant être utilisé par l'enfant pour exprimer ses conflits intrapsychiques et ses difficultés relationnelles (p. ex., Blake, 2008; Ferro, 2003; Kernberg et al., 1998). Parmi ces auteurs, Chaloner (2001) suggère que le jeu est le moyen développemental le plus approprié et puissant que possède l'enfant pour construire une relation avec l'adulte et développer sa pensée causale (comprendre les relations entre cause et effet) ainsi que ses habiletés sociales. Pour sa part, Alvarez (1988) propose que le jeu est utilisé par l'enfant pour mettre en scène, représenter, communiquer et libérer ses fantasmes inconscients, tout en lui permettant d'élaborer et de moduler les angoisses liées à ces fantasmes. Il favorise également l'expérience d'identifications anticipées, c'est-à-dire que l'enfant peut s'identifier aux personnes significatives de son entourage et élaborer ainsi différentes scènes de sa vie quotidienne. Par exemple, de retour à la maison, un enfant de 6 ans reproduit avec des figurines des situations d'intimidation qu'il a vécues récemment à l'école. Il peut d'abord s'identifier à l'agresseur, l'enfant qui induit de la peur chez les autres, puis jouer le rôle d'une victime ou d'un témoin d'un acte de violence. Ces différentes expressions ludiques lui permettent de se représenter et de s'approprier les pensées, sentiments et intentions associés aux personnes réelles ou imaginées liées au conflit relationnel mis en scène.

Winnicott (1971) a grandement contribué à la compréhension de la nature du jeu de l'enfant en proposant la notion d'espace de jeu. Selon l'auteur, l'aire où l'enfant joue n'est pas équivalente à la réalité psychique interne, puisqu'elle se situe en dehors de l'individu. Pourtant, elle n'appartient pas non plus au monde extérieur. C'est une aire d'illusion, au sens employé en psychopathologie, c'est-à-dire un espace intermédiaire entre le dedans et le dehors. En effet, lorsque l'enfant attribue une fonction ou une signification à un objet, il n'est plus l'objet réel que nous lui avons présenté; il devient un objet chargé de sens. C'est ainsi que l'enfant rassemble des objets ou des 
phénomènes appartenant à la réalité extérieure et les met au service de sa réalité interne ou personnelle (Winnicott, 1971). Selon la théorie que Winnicott (1971) a léguée, le jeu se développe graduellement au sein de la relation entre le nourrisson et sa mère ${ }^{1}$. À la naissance, le bébé ne parvient pas encore à faire la distinction entre le dedans (le moi: ce qui fait partie de lui) et le dehors (le non-moi, l'autre: ce qui constitue le monde extérieur). Lui et l'objet, qu'il soit inanimé ou humain, sont donc confondus (Winnicott, 1971). Il joue avec ses orteils comme il peut le faire avec son mobile. Ainsi, lorsqu'il suce ou mord son orteil, le bébé se rend compte que cette partie éloignée de son corps lui appartient, puisqu'il éprouve deux sensations simultanées, liées au plaisir de la succion ou au déplaisir de la morsure, à savoir la sensation dans sa bouche et celle dans son orteil, contrairement au jeu avec le mobile qui ne lui procure qu'une seule sensation. Ces petits moments de découvertes sensorielles contribuent à la différenciation entre le moi et le non-moi (ou les objets inanimés). Il en est de même lors de la tétée, qui est une source de plaisir ou de déplaisir associée à une seule sensation; l'autre appartenant à la mère. Ces autres expériences favorisent la distinction entre le moi et autrui, puisqu'à la différence de l'objet inanimé, la mère réagit aux comportements de l'enfant, à la douceur d'une tétée ou à la douleur d'une morsure. C'est aussi dans le contexte de cette relation première que la mère présente des objets au bébé. Ces objets sont acceptés, répudiés, réacceptés et, enfin, objectivement perçus par l'enfant. Par exemple, lorsque la mère lui donne un jouet, l'enfant le prend dans ses mains avec des mouvements encore imprécis, le met souvent dans sa bouche (organe permettant la reconnaissance et l'incorporation des objets durant la première année de vie), le lance, le reprend, seul ou avec l'aide de sa mère, pour enfin appréhender la nature de cet objet. Ces mouvements de va-et-vient qui se produisent entre le bébé et l'objet, grâce à la présence de la mère, contribuent à l'instauration progressive d'un sentiment de confiance et de contrôle sur l'objet (Winnicott, 1971).

Pour être capable de jouer, l'enfant a besoin d'un contexte où il se sent en sécurité. Pour ce faire, la mère se doit d'être présente et de penser à son enfant, c'est-à-dire, s'intéresser à lui et être disponible et attentive à ce qu'il fait, mais elle n'a pas à intervenir dans l'activité de jeu. Cette présence de la figure maternelle permet à l'enfant d'intérioriser une image bienveillante de celle-ci à laquelle il pourra se référer pour se sécuriser en son absence. C'est ainsi que l'enfant devient capable d'être seul, d'abord en présence de la mère et, ensuite, en son absence. Peu après, la mère commence à introduire des éléments issus de son imaginaire et découvre les réactions de 
l'enfant (Winnicott, 1971). Le chevauchement de ces deux aires de jeu, celle de l'enfant et celle de la mère, permet plus tard l'accès aux jeux partagés avec d'autres enfants. Il favorise également l'acquisition graduelle par le jeu de l'enfant d'une fonction symbolique, ce qui en permettra l'utilisation en contexte de psychothérapie. Parfois, le clinicien est témoin des jeux de l'enfant, alors qu'à d'autres moments il est invité à y prendre part. Suivant les idées de Winnicott (1971), la psychothérapie d'enfants s'effectue là où deux aires de jeu se chevauchent, celle du patient et celle du clinicien. L'auteur affirme que si le psychothérapeute ne peut pas jouer, cela signifie qu'il n'est pas fait pour ce travail. Par ailleurs, si le jeune patient n'est pas capable de jouer, au sens de faire semblant, les interventions à privilégier seront celles visant à lui permettre d'avoir accès au jeu symbolique, après quoi seulement la psychothérapie pourra commencer.

\section{Les fonctions et la valeur thérapeutique du jeu de l'enfant}

Selon Anzieu et Daymas (2007), le jeu constitue un mode créatif d'expression des enjeux intrapsychiques et relationnels qui permet à l'enfant d'acquérir les bases nécessaires au développement des habiletés cognitives (Chazan, 2002), de la communication interpersonnelle (Anzieu et Daymas, 2007), de la capacité à résoudre les problèmes (Chazan, 2002; Ferro, 2003) et de la régulation des émotions (Winnicott, 1939/1975). Le jeu donne également à l'enfant l'occasion de découvrir de nouvelles habiletés pouvant renforcer son estime de soi et la maîtrise de son environnement (Kernberg et Normandin, 2000). Dans le jeu, l'enfant peut accéder à la satisfaction de ses désirs sans pour autant s'inquiéter des conséquences sur le monde réel. Dans cette aire d'illusion, les émotions élaborées sont expérimentées par l'enfant comme étant réelles, mais elles s'avèrent moins menaçantes puisqu'elles sont représentées et ressenties dans un contexte créé par celui-ci. De cette façon, si la charge affective devient trop intense lorsque l'enfant exprime ses pulsions à travers le jeu, il peut toujours les renier ou se retirer au besoin, et y revenir éventuellement. Il n'est toutefois pas en mesure d'en faire de même dans le monde réel (Chabot et al., 2015).

En ce qui a trait à la régulation des émotions, le jeu permet entre autres à l'enfant de gérer l'agressivité qu'il peut ressentir face aux personnes de son entourage et de faire des gestes de réparation (Kernberg et Normandin, 2000). Notamment lorsque l'enfant éprouve des affects complexes ou intenses, qu'il est aux prises avec un malaise ou qu'il ressent un conflit entre des désirs contradictoires, le jeu lui permet de ventiler ce vécu 
et d'expérimenter différentes solutions, ce qui contribue à réduire la tension intrapsychique associée à ces conflits (Chabot et al., 2015). Lors d'un jeu de faire semblant, l'enfant choisit le scénario, l'histoire et les personnages mis en scène. Il attribue les différents rôles et dirige les actions. Il passe ainsi d'une position passive, soit celle qu'il détient lorsqu'il subit les conséquences d'une situation relationnelle problématique ou lorsqu'il est aux prises avec un conflit intrapsychique, à une position active, dans laquelle il regagne le contrôle de son comportement et des différents personnages imaginés mis en scène. Ceci lui permet de maîtriser davantage l'anxiété liée aux conflits qui l'habitent (Kernberg et Normandin, 2000) et de se réapproprier un certain sentiment de contrôle sur la situation à laquelle il est confronté.

Enfin, le jeu permet à l'enfant de s'identifier à ses principales figures de soins, aux personnes significatives de son entourage, ainsi qu'à des rôles sociaux importants, ce qui favorise l'acquisition des notions de règles et de limites nécessaires au fonctionnement en société (Kernberg et Normandin, 2000). Parmi celles-ci se trouvent l'interdit de l'inceste, la compréhension des différences intergénérationnelles et le respect de la propriété d'autrui. Ces identifications contribuent également au développement d'un sens de l'identité chez l'enfant (Kernberg et Normandin, 2000).

Ferro (2003) fait ressortir trois éléments qui caractérisent le jeu que l'enfant développe en contexte de psychothérapie et qui dénotent sa valeur thérapeutique. D’abord, l'auteur fait référence à sa caractéristique de nonsaturation. Selon Ferro, le jeu et les jouets permettent de multiples permutations, soit autant qu'il existe d'enfants et de conflits pouvant être représentés par ces jeux. En principe, un enfant qui se développe bien ne devrait jamais être prisonnier de la fonction induite par l'objet lui-même. Cela signifie que l'enfant peut utiliser un même jouet pour représenter différents conflits intrapsychiques ou difficultés relationnelles. Cette flexibilité lui permet également de se servir de divers jouets pour exprimer le même conflit intrapsychique ou la même difficulté relationnelle. Ensuite, Ferro suggère que le jeu, tout comme la fable, permet à l'enfant de se distancer de ses conflits, de les placer dans un ailleurs éloigné dans le temps et dans l'espace, dans des lieux mythiques et avec des personnages imaginaires (p. ex., la sorcière, un Pokémon, Link de Zelda), afin de s'identifier à ces personnages et d'expérimenter le vécu associé à la situation mise en scène. Cette caractéristique du jeu décrite par Ferro est similaire à la notion d'aire d'illusion utilisée par Winnicott (1971), selon laquelle les émotions exprimées dans le jeu appartiennent à l'enfant - puisqu'elles sont semblables aux siennes - et à la fois 
ne lui appartiennent pas - puisque, en fin de compte, il s'agit des affects des personnages impliqués dans l'activité ludique qu'il a proposée. Également, le jeu permet à l'enfant de partager ses émotions et de trouver des solutions à ses conflits puisque ce qu'il vit est arrivé à d'autres auparavant - aux personnages des histoires qu'il met en scène, ou encore aux personnages dont il s'inspire. Enfin, Ferro accorde une importance cruciale à la présence de l'autre - le psychothérapeute. Selon cet auteur, c'est la présence mentale de quelqu'un d'autre qui permettra au jeu de transformer les angoisses profondes de l'enfant. Berger (2005) appuie cette idée lorsqu'il suggère que l'enfant joue avec et pour le thérapeute. Il a besoin d'un autre sur lequel il peut projeter ses conflits, mais qui peut également lui montrer le penchant caché, nié ou moins accepté de ceux-ci. Le clinicien peut en effet repérer différents indices à travers le jeu libre de l'enfant, concernant notamment son organisation psychique, son niveau de développement, ainsi que ses états mentaux (Chazan, 2002). En permettant l'émergence d'émotions intenses et importantes de l'enfant tout en les accueillant dans un climat sécurisant, le psychothérapeute est donc en mesure de l'aider à comprendre ce qui l'habite - son monde interne - et à réguler ses affects.

\section{Le jeu normal et le jeu pathologique}

Le jeu est considéré comme un moyen d'accès à l'inconscient puisqu'il ouvre une fenêtre sur les conflits psychiques, sur la subjectivité et sur le monde de représentations de l'enfant (Kernberg et al., 1998). Dans le but de distinguer le jeu normal du jeu pathologique, Schore (2001) suggère que le niveau de jeu de l'enfant peut être représenté sur un continuum. La capacité à jouer, liée au développement normal de l'enfant, serait située à l'un des extrêmes. L'incapacité à jouer, associée au développement pathologique de l'enfant, se trouverait à l'extrême opposé.

Dans le contexte d'un développement typique, le jeu correspond à un espace sécuritaire où l'enfant peut déposer les émotions difficilement « digérables», celles dont l'intensité dépasse ses capacités de régulation affective, et où il peut explorer des pistes de solutions potentielles à ses conflits intrapsychiques ou relationnels. Les scénarios élaborés par l'enfant sont riches et il est possible d'observer une réciprocité dans les interactions entre les différents personnages mis en scène. Inversement, lors d'un développement pathologique, l'incapacité à jouer de l'enfant se manifeste par une indécision face au jeu, ainsi que par des difficultés à élaborer un scénario créatif et à utiliser le matériel de façon symbolique (Schore, 2001). Selon Kernberg 
et ses collègues (1998), l'incapacité d'un enfant à entreprendre un jeu peut être liée aux représentations qu'il s'est construites de l'autre. Ainsi, si l'enfant se représente l'adulte comme étant malveillant, il serait craintif et aurait de la difficulté à s'engager dans un jeu dans le cadre d'une psychothérapie. L'enfant doit se sentir suffisamment en sécurité dans la relation à l'autre - le psychothérapeute par exemple - avant de pouvoir s'exprimer librement dans son jeu. À mi-chemin sur le continuum se trouvent les enfants qui présentent une capacité de symbolisation pauvre et qui mettent en scène un jeu simple, désorganisé ou répétitif (Schore, 2001).

L'incapacité à jouer chez l'enfant constituerait une indication de détresse ou de psychopathologie (Chazan, 2002). Elle peut être également un indice d'exposition à un ou à plusieurs traumas au cours du développement. Certains auteurs mentionnent que les enfants maltraités tendent à mettre en scène dans leur jeu les expériences traumatiques auxquelles ils ont été confrontés (Chazan, 2004; Romano, 2010; Terr, 1990, 1991). Bien qu'il soit répétitif et moins élaboré, le jeu des enfants ayant vécu diverses formes de trauma leur permet de se réapproprier leur souffrance, mais, à la différence des autres enfants, aucun plaisir n'est procuré par l'activité ludique (Romano et al., 2008).

\section{Traumas complexes et jeu traumatique}

Lorsqu'un enfant grandit dans un environnement instable, négligent ou dangereux, il ne dispose pas des conditions optimales et ne peut compter sur les fonctions des figures parentales qui sont nécessaires au développement des capacités de symbolisation, de jeu et de faire semblant. Ainsi, les enfants d'âge préscolaire ayant vécu des mauvais traitements ou de la négligence au sein de la relation parent-enfant passent moins de temps à jouer que leurs pairs. Ils s'engagent davantage dans des jeux immatures, sensori-moteurs et fonctionnels, et moins souvent dans des jeux symboliques. De plus, les thèmes, les fantaisies et les émotions présents dans les jeux symboliques de ces enfants sont moins variés, voire restreints (Alessandri, 1991). Des observations similaires ont été effectuées auprès d'enfants victimes d'abus sexuel. Ces enfants jouent significativement moins longtemps que ceux non abusés. De plus, leurs jeux semblent être souvent interrompus de façon brusque par l'augmentation de l'anxiété associée à l'intrusion de pensées, d'affects et de souvenirs liés aux situations d'abus (Gendron, 1998).

Terr $(1990,1991)$ fut l'un des premiers auteurs à constater les spécificités du jeu des enfants ayant vécu des traumas, qu'elle désigne sous l'appellation 
de «jeu traumatique». Ce jeu est caractérisé par son intensité ainsi que par la présence d'émotions négatives et de manifestations d'anxiété qui peuvent devenir envahissantes. Les capacités de régulation affective de l'enfant ne sont plus suffisantes, ce qui l'amène à éviter ou à interrompre ses activités ludiques. Le jeu traumatique se définit également par une compulsion à la répétition (Kernberg et Normandin, 2000). Malgré les efforts conscients et inconscients de l'enfant pour éviter l'irruption du trauma, des thèmes associés à ce dernier peuvent être mis en scène d'une façon souvent rigide, répétitive et littérale (Terr, 1990, 1991). Selon Terr, ce type de jeu se présente autant chez les enfants qui ont vécu un trauma de type I (un facteur de stress unique, inattendu, limité dans le temps et vécu par plusieurs individus) que de type II (plusieurs facteurs de stress vécus de façon répétée par un seul individu).

Plus récemment, Romano (2010) suggère trois critères permettant de distinguer le jeu traumatique du jeu normatif, soit l'intensité de la souffrance psychique exprimée par l'enfant, l'absence de plaisir éprouvé lors de l'activité ludique et la déliaison des interactions intersubjectives avec le clinicien. Ce dernier critère correspond à une réaction provoquée chez le psychothérapeute par la pétrification émotionnelle de l'enfant, qui s'exprime par une altération de la capacité à penser, par une sorte de fascination ou par un déni catégorique à l'égard de ce qui est observé dans le jeu. Se basant sur les écrits de Terr (1990, 1991), l'auteure identifie trois types de jeu post-traumatique: le jeu traumatique, le jeu abréactif et la reconstitution par le jeu. Le jeu traumatique se manifeste lorsque l'enfant demeure sous l'emprise du trauma et qu'il est dans l'incapacité de s'en dégager. Ce jeu, très angoissant pour l'enfant, est automatique, souvent compulsif et exclut toute forme d'étayage. En d'autres termes, il s'agit d'une reproduction littérale du trauma vécu. Le jeu traumatique se manifeste plus fréquemment dans les situations de traumas complexes, c'est-à-dire lorsque l'enfant a été exposé, de façon répétée et prolongée dans le temps, et ce, dès un très jeune âge, à de multiples événements traumatiques survenus au sein de ses relations interpersonnelles significatives (Courtois, 2004; Herman, 1992). Lorsqu'un enfant est confronté à ce type de trauma, tel que les abus physiques ou sexuels intrafamiliaux, il ne peut pas compter sur ses figures principales de soins pour le rassurer et l'aider à élaborer les expériences traumatiques, puisqu'elles sont celles qui lui infligent les mauvais traitements. Dans ce cas, le jeu, inconsciemment associé à l'émergence possible du trauma, devient une source importante d'angoisse qui empêche toute possibilité 
d'élaboration psychique chez l'enfant. Pour sa part, le jeu abréactif représente une tentative d'élaboration et d'assimilation du trauma. L'enfant parvient à trouver différentes fins à ses histoires plutôt que de rejouer le trauma de façon littérale et compulsive. Les pensées, les émotions et les sensations physiques identifiées lors d'un jeu abréactif rappellent celles liées au trauma, mais l'enfant réussit à s'en dégager partiellement. Finalement, la reconstitution par le jeu consiste en une répétition de quelques éléments du trauma que l'on observe dans les jeux et intérêts de l'enfant. En fait, ce n'est pas le trauma dans sa globalité qui est mis en scène, mais plutôt certains aspects de celui-ci. Ce type de jeu survient lorsque l'enfant a retrouvé un certain équilibre psychique post-trauma. Ainsi, la reconstitution par le jeu n'inclut que certaines traces du vécu traumatique et la charge affective associée au jeu est moins envahissante et intense que dans les jeux traumatique et abréactif. Par conséquent, il est plus difficile de le repérer dans le jeu libre de l'enfant, les indices apparaissant plus subtils au clinicien (Romano, 2010). Le jeu abréactif et la reconstruction par le jeu laissent présumer une blessure psychique plus modérée que le jeu traumatique ou une certaine élaboration du trauma possiblement liée à la présence contenante d'un parent ayant aidé l'enfant à composer avec les effets délétères de celui-ci. Ces manifestations sont souvent associées à des traumas de type I, tels qu'un événement catastrophique ou un abus sexuel extrafamilial.

Pour sa part, Chazan (2004) élargit la notion de jeu traumatique afin d'inclure la répétition de thèmes spécifiques dans les activités ludiques de l'enfant qui s'accompagne d'affects intenses d'anxiété, de tension, ou encore de désespoir ou de tristesse. Selon l'auteure, le jeu demeure sans résolution, ou prend fin de façon abrupte ou catastrophique. Chazan accorde une importance particulière aux affects qui se manifestent lors d'un jeu traumatique. Ceux-ci seraient souvent exprimés de façon rigide et intense. Ces difficultés de régulation émotionnelle témoignent de la gravité du vécu traumatique. Chazan et Cohen (2010) proposent une nouvelle classification du jeu traumatique selon trois catégories qui révèlent les stratégies de défense et d'adaptation déployées par l'enfant lors des activités ludiques, soit la reviviscence traumatique (overwhelming re-experiencing), la reconstruction sans apaisement (re-enactment without soothing) et la reconstruction avec apaisement (re-enactment with soothing). La reviviscence traumatique consiste en un jeu chaotique où l'enfant semble complètement dépassé par les événements. Les états mentaux qui y sont exprimés sont souvent incohérents et l'histoire mise en scène est peu structurée. L'enfant manifeste des 
symptômes d'hypervigilance ou de dissociation. Il semble figé, déconnecté et tendu. La reviviscence traumatique tend ainsi à prolonger ou à aggraver l'état émotionnel dans lequel se trouve l'enfant, plutôt que de l'apaiser. Pour sa part, la reconstruction sans apaisement réfere à la répétition dans le jeu de thèmes ou de certains aspects liés au trauma. Les émotions qui y sont exprimées sont souvent préoccupantes. Par exemple, l'enfant démontre de l'agressivité tout en étant effrayé. Le récit de l'enfant est limité et rigide et ne lui apporte pas de satisfaction. Selon Chazan et Cohen, ce type de jeu ne permet pas l'élaboration du trauma, laissant l'enfant aux prises avec des sentiments de détresse et de terreur. Par ailleurs, la reconstruction avec apaisement est un jeu où l'enfant vit une expérience satisfaisante et parvient à des solutions. Bien que certains thèmes traumatiques soient mis en scène dans le jeu, l'enfant y exprime une variété d'émotions. La fin de l'activité ludique est positive et s'accompagne de sentiments de satiété, de soulagement et de maitrise chez l'enfant (Chazan et Cohen, 2010).

La présence de jeux et de thèmes traumatiques qui envahissent les activités ludiques de l'enfant ne constitue pas la seule conséquence des traumas pouvant affecter son développement et la possibilité de bénéficier d'une psychothérapie. Le développement de la capacité de mentalisation (CM) peut aussi être entravé par les traumas complexes.

\section{Effets des traumas complexes sur la capacité de mentalisation et le jeu de l'enfant}

Parmi les diverses conséquences néfastes des traumas complexes, il importe de s'attarder à l'impact qu'ils peuvent avoir sur le développement de la CM de l'enfant. Cette habileté est étroitement liée à la capacité symbolique et de faire semblant (Tessier, Normandin, Ensink et Fonagy, 2016), et elle est essentielle au travail d'élaboration psychique en contexte de psychothérapie. La mentalisation réfère à une forme d'activité mentale imaginative (Fonagy, et al., 2007), subjective et dynamique (Bateman et Fonagy, 2013), qui permet à l'individu de percevoir et d'interpréter ses propres comportements et ceux d'autrui en considérant les états mentaux sous-jacents à ces comportements, notamment les pensées, les sentiments, les attentes et les intentions (Allen et al., 2008; Fonagy et al., 2002). Dans une série d'articles phares sur le développement de la CM, Fonagy et Target (1996, 2000, 2007; Target et Fonagy, 1996) proposent que le jeu a un rôle central dans la découverte et la compréhension du monde mental de l'enfant et dans sa capacité à distinguer les réalités interne et externe. Plus précisément, le jeu de 
fantaisie est une activité mentale précoce qui permet à l'enfant de structurer son expérience subjective dans un récit cohérent, l'aidant ainsi à attribuer un sens à ses propres états mentaux et à ceux des autres (Ensink et Mayes, 2010; McMahon, 2009; Slade, 1994).

Comme le jeu, la CM se développe dans le contexte de la relation entre l'enfant et ses principales figures de soin. La mère permet à l'enfant d'acquérir des habiletés de mentalisation grâce à sa propre capacité à le considérer, dès sa naissance, comme un être humain doté d'un esprit, à réfléchir à la signification subjective des signaux qu'il émet et à traiter ces messages comme un acte intentionnel de communication (Fonagy et al., 2002).

Plusieurs chercheurs ont fait ressortir l'impact que les traumas complexes peuvent avoir sur l'habileté de l'enfant à comprendre son propre monde mental et celui d'autrui. Cicchetti et al. (2003) et Rosnay et al. (2008) ont notamment démontré que l'abus et la négligence sont associés à la présence de difficultés sociocognitives chez l'enfant, telles que des problèmes aux plans de la théorie de l'esprit ${ }^{2}$ et de la compréhension émotionnelle. D'autre part, les enfants ayant vécu des abus ou de la négligence possèdent un lexique restreint pour décrire leurs états internes (Beeghly et Cicchetti, 1994). Ensink et al. (2014) suggèrent que les enfants ayant un historique d'abus sexuel intrafamilial ou extrafamilial possèdent une CM significativement inférieure à celle d'enfants tout venant. Les résultats préliminaires d'une étude récente, effectuée par Dubé et al. (soumis) auprès d'enfants hébergés en contexte de protection de la jeunesse ayant vécu des traumas complexes, démontrent que ces enfants refusent de mentaliser ou présentent une CM qualifiée d' "absente», ce qui signifie qu'il est particulièrement difficile pour eux de se référer aux états mentaux pour se décrire et se comprendre, ainsi que pour décrire et comprendre leurs parents.

D'un point de vue clinique, Achim et Terradas (2015; Terradas et Achim, 2016) et Domon-Archambault et Terradas (2015) constatent la présence de difficultés importantes de mentalisation chez la plupart des enfants qui consultent en pédopsychiatrie, ainsi que chez ceux qui sont sous la protection de la jeunesse. Selon Terradas et al. (2016), ces enfants présentent diverses caractéristiques témoignant de tels déficits, soit un fonctionnement psychique sous le primat de l'agir et du ressenti corporel, des lacunes au plan de la régulation du comportement et des affects, des difficultés à s'intéresser et à prendre en compte leur monde interne et celui d'autrui, ainsi qu'une réflexion limitée, voire absente, quant aux états mentaux sous-jacents à leurs propres comportements et à ceux des autres. Par conséquent, ces enfants ne 
sont pas en mesure de profiter des interventions psychothérapeutiques axées sur le recours à la parole et à la symbolisation (Achim et Terradas, 2015).

Le trauma complexe empêcherait l'enfant de compléter le cycle de développement de la $\mathrm{CM}$ et ferait en sorte qu'il présenterait un fonctionnement psychique caractéristique des modes prémentalisants (Terradas et al., 2016). En réaction au trauma, l'enfant évite - inconsciemment ou volontairement - de penser aux états mentaux souffrants liés à l'expérience traumatique vécue dans la relation parent-enfant, afin de conserver des représentations positives des figures de soins abusives (Ensink et al., 2016). Suivant les idées de Fonagy et al. (2002), nous proposons que la négligence extrême, les traumas sévères, la difficulté des parents à différencier leurs états mentaux de ceux de leurs enfants et le manque, chez les parents, de sensibilité et de congruence face aux besoins physiques et émotifs exprimés par l'enfant, voire leur indifférence, ont des impacts significatifs sur le développement de la CM de l'enfant, impacts qui se lient aux difficultés de ce dernier à jouer et à faire semblant (Terradas et al., 2016). Ainsi, lorsque l'enfant est laissé à lui-même de façon répétée et que les parents ne lui offrent que très peu de rétroaction concernant son ressenti corporel et ses expériences émotives, il n'a pas l'occasion de développer des représentations mentales de ses états internes ou encore il élabore des représentations mentales peu étoffées qui demeurent très proches de ses sensations physiques (Fonagy et al., 2002). Quand l'enfant est exposé à des traumas sévères dans ses premières années de vie, il est fort probable qu'il ne tienne pas compte des états mentaux de l'autre plus tard au cours de son développement. L'enfant aura plutôt tendance à éviter d'accéder au monde mental, puisque la considération de son parent comme étant habité d'états mentaux malveillants à son égard peut provoquer une grande détresse en lui et s'avérer potentiellement traumatique (Allen, 2001; Fonagy et Target, 2000). Concernant la capacité à jouer, nous émettons l'hypothèse selon laquelle ces enfants présentent des difficultés importantes au plan de l'imagination et ne sont pas en mesure de faire semblant lorsqu'ils jouent. Cette pauvreté imaginative se traduit par une difficulté à choisir un jeu, à expliquer les activités ludiques au psychothérapeute et à transformer le matériel mis à leur disposition. L'enfant a tendance à prendre les objets pour ce qu'ils sont et est incapable de concevoir qu'ils peuvent représenter d'autres choses. Il est souvent centré sur des jeux à caractère physique impliquant la motricité fine ou grossière. Les mouvements répétitifs deviennent fréquemment la composante fondamentale du jeu, qui ne semble pas être investi au plan imaginaire (Terradas et al., 2016). 
Ces enfants ont également de la difficulté à utiliser des médiums de symbolisation (p. ex., peinture, écriture, argile) autrement que dans la décharge motrice (Verfaille, 2016).

Une difficulté chez le parent à différencier son expérience subjective et celle de l'enfant fait également obstacle au développement de la CM de ce dernier. Alors que l'un des rôles centraux du parent est de contenir les émotions de l'enfant afin que celles-ci ne dépassent pas ses capacités adaptatives, dans le contexte où cette capacité fait défaut chez le parent, l'enfant reçoit un reflet de son ressenti corporel et de ses expériences émotives qui demeure pour ainsi dire "non digéré». Par conséquent, il perçoit ses états mentaux comme contagieux et même dangereux (Leroux et Terradas, 2013). Le parent n'est donc pas en mesure d'aider son enfant à symboliser son expérience et à comprendre que celle-ci reflète sa manière d'appréhender la réalité plutôt que la réalité elle-même (Fonagy et Target, 1996). Ainsi, les distinctions moi/autre et moi/non-moi peuvent en être affectées. En ce qui a trait à la capacité à jouer, nous proposons que l'enfant confronté à ce manque de différenciation a de la difficulté à jouer avec la réalité et à faire semblant, car cela lui semble trop réel et authentique. Il évite certains jeux, de peur que ceux-ci ne lui occasionnent des conséquences dans la réalité. Ainsi, l'enfant peine à concevoir qu'il peut jouer des rôles issus de son monde imaginaire ou des expériences qu'il a vécues sans influencer le monde externe. Il a également de la difficulté à incarner un personnage et demeure souvent lui-même dans le jeu lorsqu'il dit être quelqu'un d'autre. Il peut aussi avoir tendance à poser un geste réel plutôt que de le simuler dans le contexte de jeu. Par ailleurs, suivant les idées de Ferro (2003), le jeu et les jouets perdent chez ses enfants leur caractère de non-saturation, lequel permet à l'enfant de les utiliser de façons variées et multiples. Ainsi, une fois que l'enfant attribue une signification ou une fonction - souvent la fonction réelle - à un jouet, il ne pourra pas changer d'avis, ce qui en limite l'utilisation de manière symbolique. Ce manque de flexibilité le rend incapable d'utiliser différents jouets pour exprimer un même conflit. De façon similaire, un même jouet ne pourra pas changer de fonction et représenter différents conflits. En contexte de psychothérapie, l'enfant reproduit de manière rigide des jeux avec lesquels il est familier (souvent des jeux vidéo) et ne tolère pas qu'on s'écarte du scénario ou des personnages connus dans ces jeux. Il ne ressent pas le besoin d'expliquer ses jeux au clinicien et assume que ce dernier sait ce qu'il veut faire (Terradas et al., 2016). Enfin, l'enfant peut être facilement envahi par l'utilisation des 
médiums de symbolisation, car les états internes sollicités par ces médiums sont vécus comme étant trop réels (Verfaille, 2016). Par exemple, il n'est pas rare que l'enfant se désorganise après avoir abondamment utilisé de la peinture rouge pour représenter du sang.

Enfin, lorsque les parents manquent de sensibilité et de congruence en lien avec les émotions exprimées par l'enfant, ils lui donneront des rétroactions qui ne reflètent pas son expérience subjective. Centrés sur leurs propres états mentaux, les parents sont peu attentifs et sensibles à ceux de l'enfant. Ce dernier aurait alors tendance à ne pas comprendre certains aspects de son expérience ou à développer un self étranger, c'est-à-dire un ensemble de représentations aliénantes qui se confondent avec la construction de son identité ou du self et qui l'empêchent d'avoir une image cohérente et authentique de lui-même (Fonagy et al., 2002; Slade, 2005). Concernant la capacité à jouer, nous suggérons que l'enfant, bien qu'il soit capable de faire semblant, préfère alors souvent la parole au jeu en contexte de psychothérapie. Son discours est cependant déconnecté de son expérience interne et de ce qui se passe dans son environnement immédiat. Ainsi, l'enfant peut aborder des préoccupations relatives à des thèmes d'actualité (p. ex., le réchauffement de la planète) donnant l'impression au clinicien qu'il présente une pseudo-maturité. Ces thèmes, bien qu'ils soient intéressants, l'empêchent d'aborder ses propres difficultés en contexte de psychothérapie. Lorsque l'enfant développe des jeux de faire semblant, il propose plutôt des scénarios idéalisés lui permettant inconsciemment d'éviter de projeter sur le matériel ludique ses propres conflits intrapsychiques et difficultés relationnelles. En fait, la confrontation à des éléments associés à sa réalité psychique ou environnante tend à inhiber l'imagination de l'enfant et à interrompre le jeu de faire semblant (Terradas et al., 2016).

En conclusion, les traumas complexes laissent des traces significatives dans la psyché de l'enfant, en même temps qu'ils l'empêchent de développer pleinement sa $\mathrm{CM}$, une habileté nécessaire à la compréhension de son monde interne et de celui d'autrui. Ceci souligne l'importance de bien évaluer les ressources psychologiques de l'enfant avant d'entreprendre un travail thérapeutique centré sur les séquelles des traumas qu’il a vécus.

\section{Considérations relatives à la psychothérapie auprès des enfants ayant vécu des traumas complexes}

Lorsque la psychothérapie concerne un enfant ayant vécu des traumas complexes, le clinicien doit d'abord évaluer si le problème émane de déficits 
ou de conflits, intrapsychiques ou interpersonnels. Le déficit concerne les enfants chez qui les traumas complexes ont entravé le développement de la capacité à jouer et à faire semblant ainsi que les processus de symbolisation nécessaires à l'utilisation de la communication verbale comme voie d'expression de leurs difficultés. Dans ce cas, le clinicien vise tout d'abord le développement des processus et fonctions nécessaires au travail thérapeutique des contenus traumatiques, tels que la capacité à jouer et à faire semblant, la narration et la capacité de mentalisation (Terradas et DomonArchambault, 2016). Sans se substituer aux parents, le psychothérapeute accomplit une fonction d'auxiliaire du développement de l'enfant afin de lui permettre d'avoir accès aux processus mentaux essentiels à l'élaboration des conséquences des traumas complexes.

Les enfants qui manifestent quasi exclusivement des jeux traumatiques et dont l'accès à la symbolisation est limité par l'emprise des angoisses et des autres émotions intenses reliées aux traumas complexes présentent quant à eux des difficultés au niveau des conflits. Pourtant, la perte temporaire de la capacité à jouer s'apparente à première vue à un déficit des processus mentaux soutenant la symbolisation. Dans ce contexte, le travail du clinicien consiste d'abord à rétablir la capacité à jouer de l'enfant pour ensuite l'aider à surmonter l'expérience traumatique. En d'autres termes, la psychothérapie porte initialement sur l'actualisation des processus nécessaires au travail du contenu traumatique qui, pour sa part, aura lieu dans un second temps (Terradas et Domon-Archambault, 2016). Suivant les idées de Winnicott (1939/1975), il est primordial de développer ou de restituer la capacité de l'enfant à jouer (et à faire semblant) pour qu'il soit ensuite en mesure d'élaborer psychiquement les conflits intrapsychiques et les difficultés au plan relationnel reliés aux traumas complexes.

Un dernier cas de figure concerne les enfants qui, bien qu'ils aient été exposés à ce type d'événements, ont néanmoins réussi à développer les processus mentaux permettant l'élaboration symbolique des contenus liés aux traumas grâce à la présence bienveillante d'un tiers (p. ex., une grand-mère, les membres de la fratrie, des parents d'accueil). Puisqu'ils ont suffisamment développé leur capacité de mentalisation et de faire semblant, le clinicien se centre sur la résolution des conflits intrapsychiques et relationnels pouvant être liés, évoqués ou renforcés par les événements traumatiques vécus par ces enfants. Autrement dit, le travail thérapeutique porte essentiellement sur le contenu des rencontres tel qu'il se manifeste à travers le jeu et la communication verbale de l'enfant, en utilisant les techniques d'intervention 
proposées par l'approche analytique (Terradas et Domon-Archambault, 2016).

\author{
Miguel Terradas \\ Miguel.Terradas.Carrandi@USherbrooke.ca \\ Mélissa Paquette \\ Cécilanne Lepage-Voyer \\ Vincent Domon-Archambault
}

\title{
Notes
}

1. Le terme «mère» est employé pour désigner la figure de soins principale de l'enfant.

2. Cadre conceptuel de la mentalisation, lequel est davantage centré sur le développement cognitif de la capacité à expliquer le comportement d'autrui en termes d'états mentaux (Allen et al., 2008).

\section{Références}

Achim, J. et Terradas, M. M. (2015). Le travail clinique en contexte pédopsychiatrique: l'apport de la mentalisation à la pratique d'aujourd'hui. Filigrane, 24 (2), 79-92.

Alessandri, S. M. (1991). Play and social behavior in maltreated preschoolers. Development and Psychopathology, 3 (2), 191-205.

Allen, J. G. (2001). Traumatic Relationships and Serious Mental Disorders. Chichester: John Wiley \& Sons.

Allen, J. G., Fonagy, P. et Bateman, A. W. (2008). Mentalizing in Clinical Practice. Washington, DC: American Psychiatric Publishing, Inc.

Alvarez, A. (1988). Beyond the unpleasure principle: Some preconditions for thinking through play. Journal of Child Psychotherapy, 14, 1-13.

Anzieu, A. et Daymas, S. (2007). Introduction. Dans A. Anzieu, C. Anzieu-Premmereur et S. Daymas (dir.), Le jeu en psychothérapie de l'enfant (p. 3-11). Paris, France: Dunod.

Bateman, A. W. et Fonagy, P. (2013). Mentalization based treatment. Psychoanalytic Inquiry, 33, 595-613. doi: 10.1080/07351690.2013.835170

Beeghly, M., et Cicchetti, D. (1994). Child maltreatment, attachment, and the self system: Emergence of an internal state lexicon in toddlers at high social risk. Development and Psychopathology, 6, 5-30.

Berger, M. (2005). L'enfant instable. Approche clinique et thérapeutique (2 ${ }^{\mathrm{e}}$ éd.). Paris: Dunod. Blake, P. (2008). The setting, physical and mental, and limits. Dans P. Blake. Child and Adolescent Psychotherapy (p. 193-208). Londres: Karnac.

Chabot, A., Achim, J. et Terradas, M. M. (2015). La capacité de mentalisation de l'enfant à travers le jeu et les histoires d'attachement à compléter: perspectives théorique et clinique. La psychiatrie de l'enfant, 58 (1), 207-239.

Chaloner, W. B. (2001). Counselors coaching teachers to use play therapy in classrooms: The Play and Language to Succeed (PALS) early, school-based intervention for behaviorally at-risk children. Dans A. A. Drewes, L. Carey et C. Schaefer (dir.), School-Based Play Therapy (p. 368-390). New York, NY: Wiley \& Sons. 
Chazan, S. E. (2002). Profiles of Play: Assessing and Observing Structure and Process in Play Therapy. Londres, Angleterre: Jessica Kingsley Publishers.

Chazan, S. E. (2004). Children's Play Therapy Instrument (CPTI). Manual. (Version revisée par S. E. Chazan du Children's Play Therapy Instrument (CPTI), Kernberg, P. F., Chazan, S. E., et Normandin, L. (1997)). New York, NY. Document inédit.

Chazan, S. E. et Cohen, E. (2010). Adaptative and defensive strategies in post-traumatic play of young children exposed to violent attacks. Journal of Child Psychotherapy, 36 (2), 133-151.

Cicchetti, D., Rogosch, F. A., Maughan, A., Toth, S. L. et Bruce, J. (2003). False belief understanding in maltreated children. Development and Psychopathology, 15, 1067-1091.

Courtois, C. A. (2004). Complex trauma, complex reactions: Assessment and treatment. Psychotherapy: Theory, Reasearch, Practice, Training, 41 (2), 412-425.

Domon-Archambault, V. et Terradas, M. M. (2015). Les interventions fondées sur la notion de mentalisation auprès des enfants en situation d'hébergement. Revue québécoise de psychologie, 36 (2), 1-34.

de Rosnay, M., Harris, P. L. et Pons, F. (2008). Emotion understanding and developmental psychopathology in young children. Dans C. Sharp, P. Fonagy, \& I. Goodyer (dir.), Social Cognition and Developmental Psychopathology (p. 343-386). New York, NY: Oxford University Press.

Dubé, G., Terradas, M. M. et Achim, J. (soumis). Empathie, mentalisation et comportements extériorisés chez les enfants d'âge scolaire hébergés en centre jeunesse. Revue de psychoéducation.

Ensink, K., Bégin, M., Normandin, L., Godbout, N. et Fonagy, P. (2016). Mentalization and dissociation in the context of trauma: Implications for child psychopathology. Journal of Trauma \& Dissociation. DOI: 10.1080/15299732.2016.1172536

Ensink, K. et Mayes, L. C. (2010). The development of mentalisation in children from a theory of mind perspective. Psychoanalytic Inquiry, 30 (4), 301-337.

Ensink, K., Normandin, L, Target, M., Fonagy, P., Sabourin, S. et Berthelot, N. (2014). Mentalization in children and mothers in the context of trauma: An initial study of the validity of the Child Reflective Functioning Scale. British Journal of Developmental Psychology, 33 (2), 203-217. doi: 10.1111/bjdp.12074

Ferro, A. (2003). Le jeu. Dans auteur. L'enfant et le psychanalyste (p. 81-107). Paris: Érès.

Fonagy, P., Gergely, G., Jurist, E. et Target, M. (2002). Affect Regulation, Mentalization, and the Development of the Self. New York: Other Press.

Fonagy, P., Gergely, G. et Target, M. (2007). The parent-infant dyad and the construction of the subjective self. Journal of Child Psychology and Psychiatry, 48 (3), 288-328. doi: 10.1111/j.1469-7610.2007.01727.x

Fonagy, P. et Target, M. (1996). Playing with reality I: Theory of mind and the normal development of psychic reality. International Journal of Psycho-Analysis, 77, 217-234.

Fonagy, P. et Target, M. (2000). Playing with reality III: The persistence of dual psychic reality in borderline patients. International Journal of Psycho-Analysis, 81, 853-779.

Fonagy, P. et Target, M. (2007). Playing with reality: IV. A theory of external reality rooted in intersubjectivity. International Journal of Psychoanalysis, 88 (4), 917-937.

Gendron, L. (1998). Analyse des séquelles psychologiques de l’abus sexuel à travers le jeu libre d'enfants de 2 à 7 ans. Université Laval. Mémoire de maîtrise. Repéré dans Bibliothèque et archives Canada.

Herman, J. L. (1992). Complex PTSD: A syndrome in survivors of prolonged and repeated trauma. Journal of Traumatic Stress, 5 (3), 377-391. 
Kernberg, P. F., Chazan, S. E. et Normandin, L. (1998). The Children's Play Therapy Instrument (CPTI). Description, Development, and Reliability studies. The Journal of Psychotherapy Practice and Research, 7 (3), 196-207.

Kernberg, P. F. et Normandin, L. (2000). Children Play Therapy Instrument adapted for sexually abused children. Cornell Medical School, New York Presbyterian Hospital, New York, NY. Manuscrit inédit.

Leroux, J., et Terradas, M. M. (2013). Fonction réflexive parentale et trouble réactionnel de l'attachement: perspectives théoriques. La psychiatrie de l'enfant, 56 (1), 293-315.

McMahon, L. (2009). Handbook of Play Therapy and Therapeutic Play ( $2^{\mathrm{e}}$ éd.). New York, NY: Routledge.

Romano, H. (2010). Traces du trauma dans les «jeux» d'enfants victimes d'évènements traumatiques. Le Journal des psychologues, 279, 57-61.

Romano, H., Baubet, T., Moro M. R. et Sturm, G. (2008) Le jeu chez l'enfant victime d'événements traumatiques. Annales médico psychologiques, 166, 702-710.

Schore, A. N., (2001). The effects of early relational trauma on right brain development, affect regulation, and infant mental health. Infant Mental Health Journal, 22 (1-2), 201-269.

Slade, A. (1994). Making meaning and making believe: Their role in the clinical process. Dans A. Slade \& D. Wolf (dir.), Children at play: Clinical and Developmental Approaches to Meaning and Representation (p. 81-110). Oxford, UK: Oxford University Press.

Slade, A. (2005). Parental reflective functioning: An introduction. Attachment \& Human Development, 7, 269-281.

Target, M. et Fonagy, P. (1996). Playing with reality: II. The development of psychic reality from a theoretical perspective. The International Journal of Psycho-Analysis, 77 (3), 459-479.

Terr, L. (1990). Too Scared to Cry: Psychic Trauma in Childhood. New York, NY: Harper \& Row Publishers.

Terr, L. C. (1991). Childhood traumas: An outline and overview. The American Journal of Psychiatry, 148 (1), 10-20.

Terradas, M. M. et Achim, J. (2016). Sofrimento nos laços pais-filhos: práticas educativas fundadas na mentalização na proteção da infância. Estilos da Clinica, 21 (1), 170-188.

Terradas, M. M. et Domon-Archambault, V. (2016). Conceptualisation de la psychothérapie d'enfants à la lumière des défis rencontrés en pédopsychiatrie et en protection de l'enfance. La psychiatrie de l'enfant, 59 (2), 537-574.

Terradas, M. M., Domon-Archambault, V., Achim, J. et Ensink, K. (2016). Évaluations et interventions en pédopsychiatrie: contribution de la mentalisation et de ses précurseurs à la pratique. Revue québécoise de psychologie, 37 (3), 135-157.

Tessier, V. P., Normandin, L., Ensink, K. et Fonagy, P. (2016). Fact or fiction? A longitudinal study of play and the development of reflective functioning. Bulletin of the Menninger Clinic, 80 (1), 60-79.

Verfaille, M. (2016). Mentalizing in Arts Therapies. Londres: Karnac Books.

Winnicott, D. W. (1971). Jeu et réalité: L'espace potentiel. Paris: Gallimard.

Winnicott, D. W. (1975). Pourquoi les enfants jouent-ils? Dans auteur. L'enfant et le monde extérieur (p. 123-128). Paris: Payot. Travail original publié en 1939. 